This is the peer reviewed version of the following article: Cabré, X. (2017). Isoperimetric, Sololev, and eigenvalue inequalities via the AlexandroffBakelman-Pucci method: A survey. Chinese annals of mathematics. Series B, (38), 1: 201-214." which has been published in final form at [doi: 10.1007/s11401-016-1067-0]. This article may be used for non-commercial purposes in accordance with Springer Link 


\title{
Isoperimetric, Sobolev, and Eigenvalue Inequalities via the Alexandroff-Bakelman-Pucci Method: A Survey*
}

\author{
Xavier $\mathrm{CABRE}^{1,2}$ \\ (Dedicated to Haïm Brezis, with great admiration)
}

\begin{abstract}
This paper presents the proof of several inequalities by using the technique introduced by Alexandroff, Bakelman, and Pucci to establish their ABP estimate. First, the author gives a new and simple proof of a lower bound of Berestycki, Nirenberg, and Varadhan concerning the principal eigenvalue of an elliptic operator with bounded measurable coefficients. The rest of the paper is a survey on the proofs of several isoperimetric and Sobolev inequalities using the ABP technique. This includes new proofs of the classical isoperimetric inequality, the Wulff isoperimetric inequality, and the Lions-Pacella isoperimetric inequality in convex cones. For this last inequality, the new proof was recently found by the author, Xavier Ros-Oton, and Joaquim Serra in a work where new Sobolev inequalities with weights came up by studying an open question raised by Haim Brezis.
\end{abstract}

Keywords Isoperimetric inequalities, Principal eigenvalue, Wulff shapes, ABP estimate

2010 MR Subject Classification 28A75, 35P15, 35A23, 49Q20

\section{Introduction}

In this paper, we present the proof of several inequalities by using the technique introduced by Alexandroff, Bakelman, and Pucci to establish their ABP estimate. The AlexandroffBakelman-Pucci (ABP for short) estimate is an $L^{\infty}$ bound for solutions of the Dirichlet problem associated to second order uniformly elliptic operators written in nondivergence form

$$
L u=a_{i j}(x) \partial_{i j} u+b_{i}(x) \partial_{i} u+c(x) u
$$

with bounded measurable coefficients in a domain $\Omega$ of $\mathbb{R}^{n}$. It asserts that if $\Omega$ is bounded and $c \leq 0$ in $\Omega$, then, for every function $u \in C^{2}(\Omega) \cap C(\bar{\Omega})$,

$$
\sup _{\Omega} u \leq \sup _{\partial \Omega} u+C \operatorname{diam}(\Omega)\|L u\|_{L^{n}(\Omega)},
$$

where $\operatorname{diam}(\Omega)$ denotes the diameter of $\Omega$, and $C$ is a constant depending only on the ellipticity constants of $L$ and on the $L^{n}$-norms of the coefficients $b_{i}$ (see Remark 3.1 below for its proof and $[25$, Chapter 9] for more details). The estimate was proven by the previous authors in the

\footnotetext{
Manuscript received July 14, 2015. Revised October 7, 2015.

${ }^{1}$ Universitat Politècnica de Catalunya, Departament de Matemàtiques, Diagonal 647, 08028 Barcelona, Spain.

${ }^{2}$ ICREA, Pg. Lluis Companys 23, 08010 Barcelona, Spain. E-mail: xavier.cabre@upc.edu

*This work was supported by MINECO grant MTM2014-52402-C3-1-P. The author is part of the Catalan research group 2014 SGR 1083.
} 
sixties by using a technique that in this paper we call ABP method. Both the estimate and the method have applications in several areas.

First, the ABP estimate is a basic tool in the regularity theory for fully nonlinear elliptic equations $F\left(D^{2} u\right)=0$. The ABP method is also a key ingredient in Jensen's uniqueness result for viscosity solutions. For these questions, see for instance [18]. Other applications were developed around 1994 by Berestycki, Nirenberg and Varadhan [2], who established lower bounds on the principal eigenvalue of the operator $L-c(x)$ and, as a consequence, maximum principles in "small" domains. These maximum principles are very useful (when combined with the moving planes method) to establish symmetry of positive solutions of nonlinear problems $($ see $[1,9])$.

In this paper, we give a new and simple proof (unpublished before) of the lower bound of Berestycki, Nirenberg and Varadhan [2] concerning the principal eigenvalue $\lambda_{1}=\lambda_{1}\left(L_{0}, \Omega\right)$ of the operator $L_{0}:=L-c(x)$, i.e.,

$$
L_{0} u=a_{i j}(x) \partial_{i j} u+b_{i}(x) \partial_{i} u .
$$

The bound asserts that

$$
\lambda_{1}\left(L_{0}, \Omega\right) \geq \mu|\Omega|^{-\frac{2}{n}}
$$

for some positive constant $\mu$ depending only on the ellipticity constants of $L_{0}$, the $L^{\infty}$-norms of the coefficients $b_{i}$, and an upper bound for $|\Omega|^{\frac{1}{n}}$. In particular, if one has such upper bound for $|\Omega|$, then the constant $\mu$ is independent of $|\Omega|$. As a consequence, if $|\Omega|$ tends to zero then $\lambda_{1}\left(L_{0}, \Omega\right)$ tends to infinity, by (1.2).

In contrast with theirs, our proof uses only the ABP method and does not require the Krylov-Safonov Harnack inequality. Our proof gives a slight improvement of this result by showing that $\mu$ depends in fact on the $L^{n}$-norms of the coefficients $b_{i}$ instead of the $L^{\infty}$-norms. To prove this lower bound on $\lambda_{1}$, we apply the ABP method to the problem satisfied by the logarithm of the principal eigenfunction of $L_{0}$.

Note that the constant $\mu$ in the lower bound does not depend on any modulus of regularity for the coefficients of $L_{0}$. This is why we say that it is a bound for operators with bounded measurable coefficients. This generality is crucial for the applications to fully nonlinear elliptic equations.

When $L_{0}$ is in divergence form with bounded measurable coefficients, (1.2) was proved by Brezis and Lions [5]. They established an estimate of the type (1.1) with $L^{n}$ replaced by $L^{\infty}$. Applied to the first eigenfunction, it gives (1.2) for operators in divergence form.

An improvement of the ABP estimate (1.1) in which $\operatorname{diam}(\Omega)$ is replaced by $|\Omega|^{\frac{1}{n}}$ was proved by the author in [7] (see also [9]).

When $L_{0}=\Delta$ is the Laplacian, (1.2) with its best constant $\mu$ is the Faber-Krahn inequality, and becomes an equality when $\Omega$ is a ball (see [24]). Thus, among sets with the same given volume, the ball has the smallest first Dirichlet eigenvalue. In this respect, we would like to raise the following question.

Open Problem 1.1 When $L_{0}=\Delta$ is the Laplacian, can one prove the Faber-Krahn inequality (that is, the inequality (1.2) with best constant, achieved by balls) by using an ABP method as described in the following sections?

The rest of this paper is a survey in several isoperimetric inequalities proved by using the $\mathrm{ABP}$ method. We first present the proof of the classical isoperimetric inequality in $\mathbb{R}^{n}$ found by 
the author around 1996 (see $[8,10])$. It uses the ABP technique applied to a linear Neumann problem for the Laplacian, instead of applying the method to a Dirichlet problem as in the $\mathrm{ABP}$ estimate. It yields then the isoperimetric inequality with best constant. In addition, the proof does not require the domain to be convex, and it shows easily that balls are the only smooth domains for which the equality holds.

The proof using the ABP method can also be adapted to anisotropic perimeters. This gives a new proof of the Wulff isoperimetric inequality, presented in Section 4.

The proof has also been recently extended by Serra and Teixidó [32], in a very clever way, to domains in simply connected Cartan-Hadamard Riemannian manifolds of dimension two. These are manifolds with nonpositive sectional curvature. In this way, they give a new proof that the Euclidean isoperimetric inequality (i.e., (3.1) below with the Euclidean constant $P\left(B_{1}\right) /\left|B_{1}\right|^{\frac{n-1}{n}}$ ) is also valid in such two-dimensional manifolds (with the same Euclidean constant on it). In higher dimensions (except for 3 and 4 ), this is an important conjecture which has been open for long time (see [22]).

Finally, Section 5 concerns the recent paper [16], by the author, Ros-Oton and Serra, where we established new isoperimetric and Sobolev inequalities with weights in convex cones of $\mathbb{R}^{n}$. In particular, we give a new poof of the Lions-Pacella isoperimetric inequality (see [29]) in convex cones. Let us recall that the classical proofs of the Wulff and the Lions-Pacella isoperimetric inequalities used the Brunn-Minkowski inequality (4.2).

The result in [16] states that Euclidean balls centered at the origin solve the weighted isoperimetric problem in any open convex cone $\Sigma$ of $\mathbb{R}^{n}$ (with vertex at the origin) for the following class of weights. Here, both perimeter and measure are computed with respect to the weight. The weight $w$ must be nonnegative, continuous, positively homogeneous of degree $\alpha \geq 0$, and such that $w^{\frac{1}{\alpha}}$ is concave in the cone $\Sigma$ if $\alpha>0$. This concavity condition is equivalent to a natural curvature-dimension bound, in fact, to the nonnegativeness of a BakryÉmery Ricci tensor in dimension $D=n+\alpha$. Except for the constant ones, all these weights are not radially symmetric but still balls centered at the origin are the isoperimetric sets.

Our proof uses the ABP method applied to a Neumann problem for the operator

$$
w^{-1} \operatorname{div}(w \nabla u)=\Delta u+\frac{\nabla w}{w} \cdot \nabla u .
$$

This result yields as a consequence the following Sobolev inequality. If $D=n+\alpha, 1 \leq p<D$, and $p_{*}=\frac{p D}{D-p}$, then

$$
\left(\int_{\Sigma}|u|^{p_{*}} w(x) \mathrm{d} x\right)^{\frac{1}{p_{*}}} \leq C_{w, p, n}\left(\int_{\Sigma}|\nabla u|^{p} w(x) \mathrm{d} x\right)^{\frac{1}{p}}
$$

for all smooth functions $u$ with compact support in $\mathbb{R}^{n}$ (and not necessarily vanishing on $\partial \Sigma$ ). We can give the value of the best constant $C_{w, p, n}$, since it is attained by certain radial functions (see [14]).

Monomial weights

$$
w(x)=x_{1}^{A_{1}} \cdots x_{n}^{A_{n}} \quad \text { in } \Sigma=\left\{x \in \mathbb{R}^{n}: x_{i}>0 \text { whenever } A_{i}>0\right\}
$$

(here $A_{i} \geq 0$ ) are an example of weights satisfying the above assumptions. The Sobolev inequality (1.3) with the above monomial weights $w$ appeared naturally in [13], where the author and Ros-Oton studied the following open question raised by Haim Brezis.

Open Problem 1.2 (Haim Brezis, $1996[4,6]$ ) Is the extremal solution of the problem $-\Delta u=\lambda f(u)$ in a bounded smooth domain $\Omega \subset \mathbb{R}^{n}$, with zero Dirichlet boundary conditions, 
always bounded if the dimension $n \leq 9$, and this for every positive, increasing, and convex nonlinearity $f$ ? (see $[4,6,13]$ for more details).

A stronger statement is whether the same conclusion holds for every stable solution of the Dirichlet problem for $-\Delta u=f(u)$ in $\Omega$. It was proved to be true in dimensions 2 and 3 by Nedev, in dimension 4 by the author, and in the radial case up to dimension 9 by the author and Capella (see the references in [17]). In [11], we showed that these regularity results hold essentially for any nonnegative nonlinearity $f$.

In [13], we studied this problem in convex domains with symmetry of double revolution, and we establish its validity up to dimension $n \leq 7$. If $\mathbb{R}^{n}=\mathbb{R}^{m} \times \mathbb{R}^{k}$, we say that a domain is of double revolution if it is invariant under rotations of the first $m$ variables and also under rotations of the last $k$ variables. Stable solutions depend only on the "radial" variables $s=$ $\sqrt{x_{1}^{2}+\cdots+x_{m}^{2}}$ and $t=\sqrt{x_{m+1}^{2}+\cdots+x_{n}^{2}}$. In these coordinates, the Lebesgue measure in $\mathbb{R}^{n}$ becomes $s^{m-1} t^{k-1} \mathrm{~d} s \mathrm{~d} t$. This is a monomial weight as in (1.4). In [13], to prove regularity results, we needed the above Sobolev inequalities with monomial weights, even with nonintegers $A_{i}$ in (1.4).

\section{The Principal Eigenvalue for Elliptic Operators with Bounded Measurable Coefficients}

The ABP estimate is the basic bound for subsolutions $u$ of the Dirichlet problem

$$
\begin{cases}L u \geq f & \text { in } \Omega, \\ u \leq 0 & \text { on } \partial \Omega,\end{cases}
$$

where $L$ is an elliptic operator written in nondivergence form

$$
L u=a_{i j}(x) \partial_{i j} u+b_{i}(x) \partial_{i} u+c(x) u
$$

in a domain $\Omega \subset \mathbb{R}^{n}$. We assume that $L$ is uniformly elliptic with bounded measurable coefficients, i.e., $b:=\left(b_{1}, \cdots, b_{n}\right) \in L^{\infty}(\Omega), c \in L^{\infty}(\Omega)$ and

$$
c_{0}|\xi|^{2} \leq a_{i j}(x) \xi_{i} \xi_{j} \leq C_{0}|\xi|^{2}, \quad \forall \xi \in \mathbb{R}^{n}, \quad \forall x \in \Omega
$$

for some constants $0<c_{0} \leq C_{0}$. The ABP estimate states that, if $\Omega$ is bounded, $c \leq 0$ in $\Omega$, $u \in C^{2}(\Omega) \cap C(\bar{\Omega})$ and $(2.1)$ holds, then

$$
\sup _{\Omega} u \leq C \operatorname{diam}(\Omega)\|f\|_{L^{n}(\Omega)},
$$

where $\operatorname{diam}(\Omega)$ denotes the diameter of $\Omega$ and $C$ is a constant depending only on $n, c_{0}$, and $\|b\|_{L^{n}(\Omega)}$.

The proof of the ABP estimate is explained below in Remark 3.1, after having presented in the detail ABP proof of the isoperimetric inequality.

In 1979, Krylov and Safonov used the ABP estimate and the Calderón-Zygmund cube decomposition to establish a deep result: The Harnack inequality for second order uniformly elliptic equations in nondivergence form with bounded measurable coefficients. This result allowed for the development of a regularity theory for fully nonlinear equations (see [18]).

Consider now the operator

$$
L_{0} u=(L-c(x)) u=a_{i j}(x) \partial_{i j} u+b_{i}(x) \partial_{i} u,
$$


and assume that $\Omega$ is a bounded smooth domain and that the coefficients $a_{i j}$ are smooth in $\bar{\Omega}$. In [2], Berestycki, Nirenberg and Varadhan proved the existence of a unique eigenvalue $\lambda_{1}=\lambda_{1}\left(L_{0}, \Omega\right)$ of $-L_{0}$ in $\Omega$ (the principal eigenvalue) having a positive (smooth) eigenfunction $\varphi_{1}$ (the principal eigenfunction):

$$
\begin{cases}L_{0} \varphi_{1}=-\lambda_{1} \varphi_{1} & \text { in } \Omega, \\ \varphi_{1}=0 & \text { on } \partial \Omega, \\ \varphi_{1}>0 & \text { in } \Omega .\end{cases}
$$

In addition, $\lambda_{1}$ is a simple eigenvalue and satisfies $\lambda_{1}>0$.

In [2, Theorem 2.5], Berestycki, Nirenberg and Varadhan used the Krylov-Safonov theory to establish the lower bound $\lambda_{1} \geq \mu|\Omega|^{-\frac{2}{n}}$ for some positive constant $\mu$ depending only on $n, c_{0}$, $C_{0}$, and an upper bound on $|\Omega|^{\frac{1}{n}}\|b\|_{L^{\infty}(\Omega)}$. We now give a simpler proof (unpublished before) of this lower bound by using the ABP method. We do not need to use the Krylov-Safonov theory. Our proof improves slightly the bound by showing that $\mu$ can be taken to depend on $\|b\|_{L^{n}(\Omega)}$, instead of $|\Omega|^{\frac{1}{n}}\|b\|_{L^{\infty}(\Omega)}$. More precisely, we have the following theorem.

Theorem 2.1 If $\Omega$ is bounded, the principal eigenvalue $\lambda_{1}\left(L_{0}, \Omega\right)$ of $L_{0}$ in $\Omega$ satisfies

$$
\lambda_{1}\left(L_{0}, \Omega\right) \geq \mu|\Omega|^{-\frac{2}{n}},
$$

where $\mu$ is a positive constant depending only on $n, c_{0}, C_{0}$ and $\|b\|_{L^{n}(\Omega)}$.

Proof Since $\varphi_{1}>0$ in $\Omega$, we can consider the function

$$
u=-\log \varphi_{1} .
$$

Using $\nabla u=-\varphi_{1}^{-1} \nabla \varphi_{1}$, we have

$$
\begin{cases}a_{i j} \partial_{i j} u=\lambda_{1}-b_{i} \partial_{i} u+a_{i j} \partial_{i} u \partial_{j} u & \text { in } \Omega, \\ u=+\infty & \text { on } \partial \Omega .\end{cases}
$$

We consider the lower contact set of $u$, defined by

$$
\Gamma_{u}=\{x \in \Omega: u(y) \geq u(x)+\nabla u(x) \cdot(y-x) \text { for all } y \in \bar{\Omega}\} .
$$

It is the set of points, where the tangent hyperplane to the graph of $u$ lies below $u$ in all $\bar{\Omega}$.

For every $p \in \mathbb{R}^{n}$, the minimum $\min _{\bar{\Omega}}\{u(y)-p \cdot y\}$ is achieved at an interior point of $\Omega$, since $u=+\infty$ on $\partial \Omega$ and $\Omega$ is bounded. At such a point $x$ in $\Omega$ of minimum of the function $y \mapsto u(y)-p \cdot y$, we have $x \in \Gamma_{u}$ and $p=\nabla u(x)$. It follows that

$$
\mathbb{R}^{n}=\nabla u\left(\Gamma_{u}\right) .
$$

It is interesting to visualize geometrically this proof by considering the graphs of the functions $p \cdot y+c$ for $c \in \mathbb{R}$. These are parallel hyperplanes which lie, for $c$ close to $-\infty$, below the graph of $u$. We let $c$ increase and consider the first $c$ for which there is contact or "touching" at a point $x$. It is clear that $x \notin \partial \Omega$, since $u=+\infty$ on $\partial \Omega$.

Using (2.4), we can apply the area formula to the map $p=\nabla u(x)$ for $x \in \Gamma_{u}$. Integrating in $\mathbb{R}^{n}$ a positive function $g=g(|p|)$ to be chosen later, we obtain

$$
\int_{\mathbb{R}^{n}} g(|p|) \mathrm{d} p \leq \int_{\Gamma_{u}} g(|\nabla u(x)|) \operatorname{det} D^{2} u(x) \mathrm{d} x .
$$


Note that $D^{2} u(x)$ is nonnegative definite at any point $x \in \Gamma_{u}$.

Next, we use the matrix inequality $\operatorname{det}(A B) \leq\left\{\frac{\operatorname{trace}(A B)}{n}\right\}^{n}$, which holds for every pair $A$ and $B$ of nonnegative symmetric matrices. This is a simple extension of the arithmeticgeometric means inequality. We apply it with $A=\left[a_{i j}(x)\right]$ and $B=D^{2} u(x)$ for $x \in \Gamma_{u}$. We also use

$$
\left(a_{i j} \partial_{i j} u\right)^{n} \leq C\left(\lambda_{1}^{n}+|b|^{n}|\nabla u|^{n}+|\nabla u|^{2 n}\right) \quad \text { in } \Gamma_{u},
$$

which follows from (2.3). Here and throughout the proof, $C$ denote a positive constant depending only on $n, c_{0}, C_{0}$ and $\|b\|_{L^{n}(\Omega)}$. We deduce that

$$
\begin{aligned}
\operatorname{det} D^{2} u & \leq c_{0}^{-n} \operatorname{det}\left(\left[a_{i j}\right] D^{2} u\right) \leq c_{0}^{-n}\left(\frac{\operatorname{trace}\left(\left[a_{i j}\right] D^{2} u\right)}{n}\right)^{n}=\left(n c_{0}\right)^{-n}\left(a_{i j} \partial_{i j} u\right)^{n} \\
& \leq C\left(\lambda_{1}^{n}+|b|^{n}|\nabla u|^{n}+|\nabla u|^{2 n}\right) \quad \text { in } \Gamma_{u} .
\end{aligned}
$$

Therefore, choosing $g(|p|)=\left(\lambda_{1}^{n}+|\Omega|^{-1}|p|^{n}+|p|^{2 n}\right)^{-1}$ in (2.5), we have

$$
\begin{aligned}
\int_{\mathbb{R}^{n}} \frac{\mathrm{d} p}{\lambda_{1}^{n}+|\Omega|^{-1}|p|^{n}+|p|^{2 n}} & \leq \int_{\Gamma_{u}} \frac{C\left(\lambda_{1}^{n}+|b|^{n}|\nabla u|^{n}+|\nabla u|^{2 n}\right)}{\lambda_{1}^{n}+|\Omega|^{-1}|\nabla u|^{n}+|\nabla u|^{2 n}} \mathrm{~d} x \\
& \leq C \int_{\Gamma_{u}}\left(1+|\Omega||b|^{n}\right) \mathrm{d} x \\
& \leq C\left(1+\|b\|_{L^{n}(\Omega)}^{n}\right)|\Omega| \leq C|\Omega| .
\end{aligned}
$$

On the other hand, using $\lambda_{1}^{n}+|\Omega|^{-1}|p|^{n}+|p|^{2 n} \leq \lambda_{1}^{n}+2|\Omega|^{-1}|p|^{n}$ for $|p| \leq|\Omega|^{-\frac{1}{n}}$, we see that

$$
\begin{aligned}
\int_{\mathbb{R}^{n}} \frac{\mathrm{d} p}{\lambda_{1}^{n}+|\Omega|^{-1}|p|^{n}+|p|^{2 n}} & \geq \int_{B_{|\Omega|^{-\frac{1}{n}}}} \frac{\mathrm{d} p}{\lambda_{1}^{n}+2|\Omega|^{-1}|p|^{n}} \\
& =c(n)|\Omega| \log \left(1+\frac{2|\Omega|^{-2}}{\lambda_{1}^{n}}\right) .
\end{aligned}
$$

Combining (2.6) and (2.7), we conclude $2|\Omega|^{-2} \lambda_{1}^{-n} \leq C$, which is the desired inequality.

\section{The Classical Isoperimetric Inequality}

In this section, we present a proof of the classical isoperimetric problem for smooth domains of $\mathbb{R}^{n}$ which uses the ABP technique. It was found by the author in 1996 and published in $[8,10]$. The proof establishes the following theorem.

Theorem 3.1 (Isoperimetric Inequality) Let $\Omega$ be a bounded smooth domain of $\mathbb{R}^{n}$. Then

$$
\frac{P(\Omega)}{|\Omega|^{\frac{n-1}{n}}} \geq \frac{P\left(B_{1}\right)}{\left|B_{1}\right|^{\frac{n-1}{n}}}
$$

where $B_{1}$ is the unit ball of $\mathbb{R}^{n},|\Omega|$ denotes the measure of $\Omega$, and $P(\Omega)$ the perimeter of $\Omega$. Moreover, the equality occurs in (3.1) if and only if $\Omega$ is a ball of $\mathbb{R}^{n}$.

Proof Let $u$ be a solution of the Neumann problem

$$
\begin{cases}\Delta u=\frac{P(\Omega)}{|\Omega|} & \text { in } \Omega, \\ \frac{\partial u}{\partial \nu}=1 & \text { on } \partial \Omega\end{cases}
$$


where $\Delta$ denotes the Laplace operator and $\frac{\partial u}{\partial \nu}$ the exterior normal derivative of $u$ on $\partial \Omega$. The constant $\frac{P(\Omega)}{|\Omega|}$ has been chosen so that the problem has a unique solution up to an additive constant. For these classical facts, see Example 2 in Section 10.5 of [27], or the end of Section 6.7 of [25]. In addition, we have that $u$ is smooth in $\bar{\Omega}$.

We consider the lower contact set of $u$, defined by

$$
\Gamma_{u}=\{x \in \Omega: u(y) \geq u(x)+\nabla u(x) \cdot(y-x) \text { for all } y \in \bar{\Omega}\} .
$$

It is the set of points, where the tangent hyperplane to the graph of $u$ lies below $u$ in all $\bar{\Omega}$. We claim that

$$
B_{1}(0) \subset \nabla u\left(\Gamma_{u}\right),
$$

where $B_{1}(0)=B_{1}$ denotes the unit ball of $\mathbb{R}^{n}$ with center 0 .

To show (3.4), take any $p \in \mathbb{R}^{n}$ satisfying $|p|<1$. Let $x \in \bar{\Omega}$ be a point, such that

$$
\min _{y \in \bar{\Omega}}\{u(y)-p \cdot y\}=u(x)-p \cdot x
$$

(this is, up to a sign, the Legendre transform of $u$ ). If $x \in \partial \Omega$, then the exterior normal derivative of $u(y)-p \cdot y$ at $x$ would be nonpositive and hence $\left(\frac{\partial u}{\partial \nu}\right)(x) \leq|p|<1$, a contradiction with (3.2). It follows that $x \in \Omega$ and, therefore, that $x$ is an interior minimum of the function $u(y)-p \cdot y$. In particular, $p=\nabla u(x)$ and $x \in \Gamma_{u}$. Claim (3.4) is now proved. It is interesting to visualize geometrically the proof of the claim, by considering the graphs of the functions $p \cdot y+c$ for $c \in \mathbb{R}$. These are parallel hyperplanes which lie, for $c$ close to $-\infty$, below the graph of $u$. We let $c$ increase and consider the first $c$ for which there is contact or "touching" at a point $x$. It is clear geometrically that $x \notin \partial \Omega$, since $|p|<1$ and $\frac{\partial u}{\partial \nu}=1$ on $\partial \Omega$.

Next, from (3.4), we deduce

$$
\left|B_{1}\right| \leq\left|\nabla u\left(\Gamma_{u}\right)\right|=\int_{\nabla u\left(\Gamma_{u}\right)} \mathrm{d} p \leq \int_{\Gamma_{u}} \operatorname{det} D^{2} u(x) \mathrm{d} x .
$$

We have applied the area formula to the map $\nabla u: \Gamma_{u} \rightarrow \mathbb{R}^{n}$, and we have used that its Jacobian, $\operatorname{det} D^{2} u$, is nonnegative in $\Gamma_{u}$ by definition of this set.

Finally, we use the arithmetic-geometric means inequality applied to the eigenvalues of $D^{2} u(x)$ (which are nonnegative numbers for $x \in \Gamma_{u}$ ). We obtain

$$
\operatorname{det} D^{2} u \leq\left(\frac{\Delta u}{n}\right)^{n} \quad \text { in } \Gamma_{u} .
$$

This, combined with $(3.5)$ and $\Delta u \equiv \frac{P(\Omega)}{|\Omega|}$, gives

$$
\left|B_{1}\right| \leq\left(\frac{P(\Omega)}{n|\Omega|}\right)^{n}\left|\Gamma_{u}\right| \leq\left(\frac{P(\Omega)}{n|\Omega|}\right)^{n}|\Omega| .
$$

Since $P\left(B_{1}\right)=n\left|B_{1}\right|$, we conclude the isoperimetric inequality

$$
\frac{P\left(B_{1}\right)}{\left|B_{1}\right|^{\frac{n-1}{n}}}=n\left|B_{1}\right|^{\frac{1}{n}} \leq \frac{P(\Omega)}{|\Omega|^{\frac{n-1}{n}}} .
$$

Note that when $\Omega=B_{1}$, then $u(x)=\frac{|x|^{2}}{2}$ and, in particular, all the eigenvalues of $D^{2} u(x)$ are equal. Therefore, it is clear that (3.4) and (3.6) are equalities when $\Omega=B_{1}$. This explains why the proof gives the isoperimetric inequality with best constant. 
The previous proof can also be used to show that balls are the only smooth domains for which equality occurs in the isoperimetric inequality. Indeed, if (3.8) is an equality, then all the inequalities in (3.5)-(3.7) are also equalities. In particular, we have $\left|\Gamma_{u}\right|=|\Omega|$. Since $\Gamma_{u} \subset \Omega$, $\Omega$ is an open set, and $\Gamma_{u}$ is closed relatively to $\Omega$, we deduce that $\Gamma_{u}=\Omega$.

Recall that the geometric and arithmetic means of $n$ nonnegative numbers are equal if and only if these $n$ numbers are all equal. Hence, the equality in (3.6) and the fact that $\Delta u$ is constant in $\Omega$ give that $D^{2} u=a \mathrm{I}$ in all $\Gamma_{u}=\Omega$, where $\mathrm{I}$ is the identity matrix and $a=\frac{P(\Omega)}{n|\Omega|}$ is a positive constant. Let $x_{0} \in \Omega$ be any given point. Integrating $D^{2} u=a \mathrm{I}$ on segments from $x_{0}$, we deduce that

$$
u(x)=u\left(x_{0}\right)+\nabla u\left(x_{0}\right) \cdot\left(x-x_{0}\right)+\frac{a}{2}\left|x-x_{0}\right|^{2}
$$

for $x$ in a neighborhood of $x_{0}$. In particular, $\nabla u(x)=\nabla u\left(x_{0}\right)+a\left(x-x_{0}\right)$ in such a neighborhood, and hence the map $\nabla u-a \mathrm{I}$ is locally constant. Since $\Omega$ is connected, we deduce that this map is indeed a constant, say $\nabla u-a \mathrm{I} \equiv y_{0}$.

It follows that $\nabla u\left(\Gamma_{u}\right)=\nabla u(\Omega)=y_{0}+a \Omega$. By (3.4), we know that $B_{1}(0) \subset \nabla u\left(\Gamma_{u}\right)=y_{0}+$ $a \Omega$. In addition, these two open smooth sets, $B_{1}(0)$ and $y_{0}+a \Omega$, have the same measure, since equality occurs in the first inequality of (3.5). We conclude that $B_{1}(0)=\nabla u\left(\Gamma_{u}\right)=y_{0}+a \Omega$, and hence that $\Omega$ is a ball.

The previous proof is also suited for a quantitative version as we will show in [12] with Cinti, Pratelli, Ros-Oton and Serra.

Remark 3.1 The ABP estimate (2.2) is proved by proceeding as in the previous proof for the isoperimetric inequality, but now by considering the Dirichlet problem (2.1) instead of (3.2). The main claim (3.4) is now replaced by $B_{\frac{M}{d}}(0) \subset \nabla u\left(\Gamma^{u}\right)$, where $M=\sup _{\Omega} u, d=\operatorname{diam}(\Omega)$ and $\Gamma^{u}$ is now the upper contact set of $u$ (see [25, Chapter 9] for details).

In 1994 (before our proof), Trudinger [35] gave a proof of the classical isoperimetric inequality by using the Monge-Ampère operator and the ABP estimate. His proof consists of applying the ABP estimate to the problem

$$
\begin{cases}\operatorname{det} D^{2} u=\chi_{\Omega} & \text { in } B_{R}, \\ u=0 & \text { on } \partial B_{R}\end{cases}
$$

where $\chi_{\Omega}$ is the characteristic function of $\Omega$ and $B_{R}=B_{R}(0)$, and then letting $R \rightarrow \infty$.

Before the proofs in [8, 35] by using ABP, there was already Gromov's proof (see [26]) of the isoperimetric inequality, which used the Knothe map (see also [19] for a presentation). A more classical proof of the isoperimetric problem is based on Steiner symmetrization (see [3, $23,31]$ ). A fifth proof consists of deducing easily the isoperimetric inequality from the BrunnMinkowski inequality (4.2) (see [24]). Finally, in 2004, Cordero-Erausquin, Nazaret and Villani [20] used the Brenier map from optimal transportation to give another proof of the isoperimetric inequality. This optimal transport proof, as well as the Knothe-Gromov one, both lead also to the Wulff isoperimetric inequality for anisotropic perimeters, which is discussed in the following section.

\section{The Wulff Isoperimetric Inequality}

In a personal communication, Robert McCann pointed out that our proof of Theorem 3.1 also establishes the following inequality concerning Wulff shapes and surface energies of crystals. 
Given any positive and smooth function $H$ on $\mathbb{S}^{n-1}=\partial B_{1}$ (the surface tension), consider the convex set $W \subset \mathbb{R}^{n}$ (called the Wulff shape) defined by

$$
W=\left\{p \in \mathbb{R}^{n}: p \cdot \nu<H(\nu) \text { for all } \nu \in \mathbb{S}^{n-1}\right\} .
$$

Note that $W$ is an open set with $0 \in W$. To visualize $W$, it is useful to note that it is the intersection of the half-spaces $\{p \cdot \nu<H(\nu)\}$ among all $\nu \in \mathbb{S}^{n-1}$. In particular, $W$ is a convex set.

For every smooth domain $\Omega \in \mathbb{R}^{n}$ (not necessarily convex), define

$$
P_{H}(\Omega):=\int_{\partial \Omega} H(\nu(x)) \mathrm{d} S(x)
$$

to be its surface energy, where $\mathrm{d} S(x)$ denotes the area element on $\partial \Omega$ and $\nu(x)$ is the unit exterior normal to $\partial \Omega$ at $x$. Then, among sets $\Omega$ with measure $|W|$, the surface energy $P_{H}(\Omega)$ is minimized by (and only by) the Wulff shape $W$ and its translates. Equivalently, for every $\Omega$ (without restriction on its measure) we have the following theorem.

Theorem 4.1 ([33-34,37]) Let $\Omega$ be a bounded smooth domain of $\mathbb{R}^{n}$. Then

$$
\frac{P_{H}(\Omega)}{|\Omega|^{\frac{n-1}{n}}} \geq \frac{P_{H}(W)}{|W|^{\frac{n-1}{n}}}
$$

with equality if and only if $\Omega=a W+b$ for some $a>0$ and $b \in \mathbb{R}^{n}$.

This theorem was first stated, without proof, by Wulff [37] in 1901. His work was followed by Dinghas [21], who studied the problem within the class of convex polyhedra. He used the Brunn-Minkowski inequality

$$
|A+B|^{\frac{1}{n}} \geq|A|^{\frac{1}{n}}+|B|^{\frac{1}{n}}
$$

valid for all nonempty measurable sets $A$ and $B$ of $\mathbb{R}^{n}$ for which $A+B$ is also measurable (see [24] for more information on this inequality). Some years later, Taylor [33-34] finally proved the theorem among sets of finite perimeter (see [16] for more references in this subject). As mentioned in the previous section, this anisotropic isoperimetric inequality also follows easily by using the Knothe-Gromov map or the Brenier map from optimal transport. In addition, a proof of the Wulff theorem using an anisotropic rearrangement was given by Van Schaftingen in [36] (with a method coming from Klimov [28]).

This anisotropic isoperimetric problem can be solved with the same method that we have used above for the isoperimetric problem. One considers now the solution of

$$
\begin{cases}\Delta u=\frac{P_{H}(\Omega)}{|\Omega|} & \text { in } \Omega, \\ \frac{\partial u}{\partial \nu}=H(\nu) & \text { on } \partial \Omega .\end{cases}
$$

Claim (3.4) is now replaced by $W \subset \nabla u\left(\Gamma_{u}\right)$, which is proved again by using the Legendre transform of $u$. Then, the area formula gives $|W| \leq\left\{\frac{P_{H}(\Omega)}{n|\Omega|}\right\}^{n}|\Omega|$.

To conclude, one uses $P_{H}(W)=n|W|$. This last equality follows from the fact that $H(\nu(p))=p \cdot \nu(p)$ for almost every $p \in \partial W$ (here $\nu(p)$ denotes the unit exterior normal to $\partial W$ at $p)$, and thus

$$
P_{H}(W)=\int_{\partial W} H(\nu(x)) \mathrm{d} S=\int_{\partial W} x \cdot \nu(x) \mathrm{d} S=\int_{W} \operatorname{div}(x) \mathrm{d} x=n|W| .
$$

A similar argument as in the previous section shows that the equality is only achieved by the sets $\Omega=a W+b$ (see [16] for details). 


\section{Weighted Isoperimetric and Sobolev Inequalities in Convex Cones}

The isoperimetric inequality in convex cones of Lions and Pacella reads as follows.

Theorem $5.1([29])$ Let $\Sigma$ be an open convex cone in $\mathbb{R}^{n}$ with vertex at 0 , and $B_{1}:=B_{1}(0)$. Then

$$
\frac{P(\Omega ; \Sigma)}{|\Omega \cap \Sigma|^{\frac{n-1}{n}}} \geq \frac{P\left(B_{1} ; \Sigma\right)}{\left|B_{1} \cap \Sigma\right|^{\frac{n-1}{n}}}
$$

for every measurable set $\Omega \subset \mathbb{R}^{n}$ with $|\Omega \cap \Sigma|<\infty$. Here $P(\Omega ; \Sigma)$ is the perimeter of $\Omega$ relative to $\Sigma$. When $\Omega$ is smooth, it agrees with the $(n-1)$-Hausdorff measure of $\partial \Omega \cap \Sigma$.

Note that $\Sigma$ is an open set. Hence, if there is a part of $\partial \Omega$ contained in $\partial \Sigma$, then it is not counted in this perimeter. The assumption of convexity of the cone can not be removed as shown in [29].

The proof of Theorem 5.1 given in [29] is based on the Brunn-Minkowski inequality (4.2). Alternatively, Theorem 5.1 can also be deduced from a degenerate case of the classical Wulff inequality of Section 4. For this, one must allow the surface energy $H$ to vanish in part of $\mathbb{S}^{n-1}$. More precisely, we say that a function $H$ defined in $\mathbb{R}^{n}$ is a gauge when

$H$ is nonnegative, positively homogeneous of degree one, and convex.

The Wulff inequality can be proved for such surface energies $H$. With this in hand, one can establish the Lions-Pacella inequality as follows.

It is easy to prove that the convex set $B_{1} \cap \Sigma$ is equal to the Wulff shape $W$, defined by (4.1), for a unique gauge $H$ (which depends on the cone $\Sigma$ ). This function $H$ vanishes on normal vectors to $\partial \Sigma$ and agrees with 1 on unit vectors inside $\Sigma$. This is why one can recover the Lions-Pacella inequality from the Wulff one associated to this $H$. In particular, the Lions-Pacella inequality can be proved by using the ABP method (see [16] for more details).

Let us now turn to the extension of the Lions-Pacella theorem in [16] to the case of some homogeneous weights, as explained in the introduction. Given a gauge $H$ and a nonnegative function $w$ defined in $\bar{\Sigma}$, consider the weighted anisotropic perimeter

$$
P_{w, H}(\Omega ; \Sigma):=\int_{\partial \Omega \cap \Sigma} H(\nu(x)) w(x) \mathrm{d} S
$$

(defined in this way when $\partial \Omega$ is regular enough) and the weighted measure

$$
w(\Omega \cap \Sigma):=\int_{\Omega \cap \Sigma} w(x) \mathrm{d} x .
$$

Theorem 5.2 ([16]) Let $H$ be a gauge in $\mathbb{R}^{n}$, i.e., a function satisfies (5.1), and $W$ with its associated Wulff shape is defined by (4.1). Let $\Sigma$ be an open convex cone in $\mathbb{R}^{n}$ with vertex at the origin, such that $W \cap \Sigma \neq \varnothing$. Let $w$ be a continuous function in $\Sigma$, positive in $\Sigma$, and positively homogeneous of degree $\alpha \geq 0$. Assume in addition that $w^{\frac{1}{\alpha}}$ is concave in $\Sigma$ in case $\alpha>0$.

Then, for each measurable set $\Omega \subset \mathbb{R}^{n}$ with $w(\Omega \cap \Sigma)<\infty$,

$$
\frac{P_{w, H}(\Omega ; \Sigma)}{w(\Omega \cap \Sigma)^{\frac{D-1}{D}}} \geq \frac{P_{w, H}(W ; \Sigma)}{w(W \cap \Sigma)^{\frac{D-1}{D}}},
$$

where $D=n+\alpha$. 
After announcing our result in [15] and posting the preprint [16], Milman and Rotem [30] found an alternative proof of our isoperimetric inequality, Theorem 5.2 ([30] mentions that the same was found independently by Nguyen). Their proof uses the Borell-Brascamp-Lieb extension of the the Brunn-Minkowski inequality.

Our key hypothesis that $w^{\frac{1}{\alpha}}$ is a concave function is equivalent to a natural curvaturedimension bound, in fact, to the nonnegativeness of a Bakry-Émery Ricci tensor in dimension $D=n+\alpha$. This was pointed out by C. Villani.

Note that the shape of the minimizer is $W \cap \Sigma$, and that $W$ depends only on $H$ and not on the weight $w$ neither on the cone $\Sigma$. In particular, in the isotropic case $H=\|\cdot\|_{2}$, we find the following noteworthy fact. Even if the weights that we consider are not radial (unless $w \equiv$ constant), still Euclidean balls centered at the origin (intersected with the cone) minimize this isoperimetric quotient.

The equality in (5.2) holds whenever $\Omega \cap \Sigma=r W \cap \Sigma$, where $r$ is any positive number. That $r W \cap \Sigma$ is the unique minimizer of (5.2) will be shown in the upcoming paper [12], where in addition we show a quantitative version of (5.2).

Note also that we allow $w$ to vanish somewhere (or everywhere) on $\partial \Sigma$. This happens in the case of the monomial weights (1.4), for which the previous theorem holds. From (5.2), it is simple to deduce the sharp Sobolev inequality with monomial weights (1.3) stated in the introduction.

Next, to show the key ideas in a simpler situation, we prove Theorem 5.2 in the isotropic case $H=\|\cdot\|_{2}$ when the weight $w \equiv 0$ on $\partial \Sigma$. This is the case of the monomial weights. To simplify, we also assume that $\Omega=U \cap \Sigma$, where $U$ is some bounded smooth domain in $\mathbb{R}^{n}$.

Let $w$ be a positive homogeneous function of degree $\alpha>0$ in an open convex cone $\Sigma \subset \mathbb{R}^{n}$. In the proof we will need an easy lemma stating that $w^{\frac{1}{\alpha}}$ is concave in $\Sigma$ if and only if

$$
\alpha\left(\frac{w(z)}{w(x)}\right)^{\frac{1}{\alpha}} \leq \frac{\nabla w(x) \cdot z}{w(x)}
$$

holds for each $x, z \in \Sigma$ (see [16]).

To prove the result, we also need the following equality. Here we denote $P_{w, H}$ by $P_{w}$, since $H$ is the Euclidean norm. Using that $w \equiv 0$ on $\partial \Sigma$, we deduce

$$
\begin{aligned}
P_{w}(W ; \Sigma) & =\int_{\partial W \cap \Sigma} H(\nu(x)) w(x) \mathrm{d} S=\int_{\partial W \cap \Sigma} x \cdot \nu(x) w(x) \mathrm{d} S \\
& =\int_{\partial(W \cap \Sigma)} x \cdot \nu(x) w(x) \mathrm{d} S=\int_{W \cap \Sigma} \operatorname{div}(x w(x)) \mathrm{d} x \\
& =\int_{W \cap \Sigma}\{n w(x)+x \cdot \nabla w(x)\} \mathrm{d} x=\int_{W \cap \Sigma}(n+\alpha) w(x) \mathrm{d} x=D w(W \cap \Sigma),
\end{aligned}
$$

where we have used that $x \cdot \nabla w(x)=\alpha w(x)$, since $w$ is homogeneous of degree $\alpha$.

A key point in the following proof is that, when $\Omega=B_{1} \cap \Sigma$, the function $u(x)=\frac{|x|^{2}}{2}$ solves $w^{-1} \operatorname{div}(w \nabla u)=b$ for some constant $b$, the normal derivative of $u$ on $\partial B_{1} \cap \Sigma$ is identically one, and the normal derivative of $u$ on $\partial \Sigma \cap B_{1}$ is identically zero.

Proof of Theorem 5.2 (In the case $w \equiv 0$ on $\partial \Sigma$ and $H=\|\cdot\|_{2}$ ) For the sake of simplicity, we assume here that $\Omega=U \cap \Sigma$, where $U$ is some bounded smooth domain in $\mathbb{R}^{n}$.

Observe that since $\Omega=U \cap \Sigma$ is piecewise Lipschitz, and $w \equiv 0$ on $\partial \Sigma$, it holds

$$
P_{w}(\Omega ; \Sigma)=\int_{\partial \Omega} w(x) \mathrm{d} x .
$$


Hence, using $w \in C(\bar{\Sigma})$ and (5.5), it is immediate to prove that for any $y \in \Sigma$, we have

$$
\lim _{\delta \downarrow 0} P_{w}(\Omega+\delta y ; \Sigma)=P_{w}(\Omega ; \Sigma), \quad \lim _{\delta \downarrow 0} w(\Omega+\delta y)=w(\Omega) .
$$

We denote $\Omega+\delta y=\{x+\delta y, x \in \Omega\}$. Note that $P_{w}(\Omega+\delta y ; \Sigma)$ could not converge to $P_{w}(\Omega ; \Sigma)$ as $\delta \downarrow 0$, if $w$ did not vanish on the boundary of the cone $\Sigma$.

By this approximation property and a subsequent regularization of $\Omega+\delta y$ (a detailed argument can be found in [16]), we see that it suffices to prove (5.2) for smooth domains whose closure is contained in $\Sigma$. Thus, from now on in the proof, $\Omega$ is a smooth domain satisfying $\bar{\Omega} \subset \Sigma$. in $\bar{\Omega}$.

At this stage, it is clear that by approximating $\left.w\right|_{\bar{\Omega}}$, we can assume $w \in C^{\infty}(\bar{\Omega})$ and $w>0$

Let $u$ be a solution of the linear Neumann problem

$$
\begin{cases}w^{-1} \operatorname{div}(w \nabla u)=b_{\Omega} & \text { in } \Omega, \\ \frac{\partial u}{\partial \nu}=1 & \text { on } \partial \Omega .\end{cases}
$$

The Fredholm alternative ensures that there exists a solution of (5.6) (which is unique up to an additive constant) if and only if the constant $b_{\Omega}$ is given by

$$
b_{\Omega}=\frac{P_{w}(\Omega ; \Sigma)}{w(\Omega)} .
$$

Note also that since $w$ is positive and smooth in $\bar{\Omega},(5.6)$ is a uniformly elliptic problem with smooth coefficients. Thus, $u \in C^{\infty}(\bar{\Omega})$. For these classical facts, see Example 2 in Section 10.5 of [27], or the end of Section 6.7 of [25].

Consider now the lower contact set of $u, \Gamma_{u}$, defined by (3.3) as the set of points in $\Omega$ at which the tangent hyperplane to the graph of $u$ lies below $u$ in all $\bar{\Omega}$. Then, as in Section 3 , we touch by below the graph of $u$ with hyperplanes of fixed slope $p \in B_{1}$, and by using the boundary condition in (5.6), we deduce that $B_{1} \subset \nabla u\left(\Gamma_{u}\right)$. From this, we obtain

$$
B_{1} \cap \Sigma \subset \nabla u\left(\Gamma_{u}\right) \cap \Sigma
$$

and thus

$$
\begin{aligned}
w\left(B_{1} \cap \Sigma\right) & \leq \int_{\nabla u\left(\Gamma_{u}\right) \cap \Sigma} w(p) \mathrm{d} p \leq \int_{\Gamma_{u} \cap(\nabla u)^{-1}(\Sigma)} w(\nabla u(x)) \operatorname{det} D^{2} u(x) \mathrm{d} x \\
& \leq \int_{\Gamma_{u} \cap(\nabla u)^{-1}(\Sigma)} w(\nabla u)\left(\frac{\Delta u}{n}\right)^{n} \mathrm{~d} x .
\end{aligned}
$$

We apply the area formula to the smooth map $\nabla u: \Gamma_{u} \rightarrow \mathbb{R}^{n}$ and also the classical arithmeticgeometric means inequality, where all eigenvalues of $D^{2} u$ are nonnegative in $\Gamma_{u}$ by definition of this set.

Next we use that, when $\alpha>0$,

$$
s^{\alpha} t^{n} \leq\left(\frac{\alpha s+n t}{\alpha+n}\right)^{\alpha+n} \text { for all } s>0, t>0,
$$

which follows from the concavity of the logarithm function. Using also (5.3), we find

$$
\frac{w(\nabla u)}{w(x)}\left(\frac{\Delta u}{n}\right)^{n} \leq\left(\frac{\alpha\left(\frac{w(\nabla u)}{w(x)}\right)^{\frac{1}{\alpha}}+\Delta u}{\alpha+n}\right)^{\alpha+n} \leq\left(\frac{\frac{\nabla w(x) \cdot \nabla u}{w(x)}+\Delta u}{D}\right)^{D} .
$$


Recall that $D=n+\alpha$. Thus, using the equation in (5.6), we obtain

$$
\frac{w(\nabla u)}{w(x)}\left(\frac{\Delta u}{n}\right)^{n} \leq\left(\frac{b_{\Omega}}{D}\right)^{D} \quad \text { in } \Gamma_{u} \cap(\nabla u)^{-1}(\Sigma) .
$$

If $\alpha=0$, then $w \equiv 1$, and (5.9) is trivial.

Therefore, since $\Gamma_{u} \subset \Omega$, combining (5.8) and (5.9), we obtain

$$
\begin{aligned}
w\left(B_{1} \cap \Sigma\right) & \leq \int_{\Gamma_{u} \cap(\nabla u)^{-1}(\Sigma)}\left(\frac{b_{\Omega}}{D}\right)^{D} w(x) \mathrm{d} x=\left(\frac{b_{\Omega}}{D}\right)^{D} w\left(\Gamma_{u} \cap(\nabla u)^{-1}(\Sigma)\right) \\
& \leq\left(\frac{b_{\Omega}}{D}\right)^{D} w(\Omega)=D^{-D} \frac{P_{w}(\Omega ; \Sigma)^{D}}{w(\Omega)^{D-1}} .
\end{aligned}
$$

In the last equality, we use the value of the constant $b_{\Omega}$, given by (5.7).

Finally, by (5.4), we have $P_{w}\left(B_{1} \cap \Sigma ; \Sigma\right)=D w\left(B_{1} \cap \Sigma\right)$. Then we obtain the desired inequality (5.2).

\section{References}

[1] Berestycki, H. and Nirenberg, L., On the method of moving planes and the sliding method, Bol. Soc. Brasil. Mat. (N.S.), 22, 1991, 1-37.

[2] Berestycki, H., Nirenberg, L. and Varadhan, S. R. S., The principal eigenvalue and maximum principle for second-order elliptic operators in general domains, Comm. Pure Appl. Math., 47, 1994, 47-92.

[3] Besson, G., From isoperimetric inequalities to heat kernels via symmetrisation, Surveys in Differential Geometry, Vol. IX, 27-51, Surv. Differ. Geom., 9, Int. Press, Somerville, MA, 2004.

[4] Brezis, H., Is there failure of the inverse function theorem? Morse theory, minimax theory and their applications to nonlinear differential equations, Proc. Workshop held at the Chinese Acad. of Sciences, Beijing, 1999, 23-33, New Stud. Adv. Math., 1, Int. Press, Somerville, MA, 2003.

[5] Brezis, H. and Lions, P.-L., An estimate related to the strong maximum principle, Boll. Un. Mat. Ital. A, 17(5), 1980, 503-508.

[6] Brezis, H. and Vázquez, J. L., Blow-up solutions of some nonlinear elliptic problems, Rev. Mat. Univ. Complut. Madrid, 10, 1997, 443-469.

[7] Cabré, X., On the Alexandroff-Bakelman-Pucci estimate and the reversed Hölder inequality for solutions of elliptic and parabolic equations, Comm. Pure Appl. Math., 48, 1995, 539-570.

[8] Cabré, X., Partial differential equations, geometry, and stochastic control (in Catalan), Butl. Soc. Catalana Mat., 15, 2000, 7-27.

[9] Cabré, X., Topics in regularity and qualitative properties of solutions of nonlinear elliptic equations, Discrete Contin. Dyn. Syst., 8, 2002, 331-359.

[10] Cabré, X., Elliptic PDEs in probability and geometry, symmetry and regularity of solutions, Discrete Contin. Dyn. Syst., 20, 2008, 425-457.

[11] Cabré, X., Regularity of minimizers of semilinear elliptic problems up to dimension four, Comm. Pure Appl. Math., 63, 2010, 1362-1380.

[12] Cabré, X., Cinti, E., Pratelli, A., et al., Quantitative isoperimetric inequalities with homogeneous weights, in preparation.

[13] Cabré, X. and Ros-Oton, X., Regularity of stable solutions up to dimension 7 in domains of double revolution, Comm. Partial Differential Equations, 38, 2013, 135-154.

[14] Cabré, X. and Ros-Oton, X., Sobolev and isoperimetric inequalities with monomial weights, J. Differential Equations, 255, 2013, 4312-4336.

[15] Cabré, X., Ros-Oton, X. and Serra, J., Euclidean balls solve some isoperimetric problems with nonradial weights, C. R. Math. Acad. Sci. Paris, 350, 2012, 945-947.

[16] Cabré, X., Ros-Oton, X. and Serra, J., Sharp isoperimetric inequalities via the ABP method, J. Eur. Math. Soc., 18, 2016, 2971-2998. 
[17] Cabré, X., Sanchón, M. and Spruck, J., A priori estimates for semistable solutions of semilinear elliptic equations, Discrete Contin. Dyn. Syst., Series A, 36, 2016, 601-609.

[18] Caffarelli, L. A. and Cabré, X., Fully Nonlinear Elliptic Equations, Colloquium Publications, 43, American Mathematical Society, Providence, RI, 1995.

[19] Chavel, I., Riemannian Geometry: A Modern Introduction, 2nd Revised Edition, Cambridge University Press, Cambridge, 2006.

[20] Cordero-Erausquin, C., Nazaret, B. and Villani, C., A mass transportation approach to sharp Sobolev and Gagliardo-Nirenberg inequalities, Adv. Math., 182, 2004, 307-332.

[21] Dinghas, A., Über einen geometrischen satz von Wulff für die gleichgewichtsform von kristallen, Zeitschrift für Kristallographie, 105, 1944, 304-314.

[22] Druet, O., Isoperimetric inequalities on nonpositively curved spaces, Lecture Notes. http://math.arizona. edu/ dido/presentations/Druet-Carthage.pdf

[23] Fusco, N., The stability of the isoperimetric inequality, CNA Summer School, Carnegie Mellon University, Pittsburgh, 2013.

[24] Gardner, R. J., The Brunn-Minkowski inequality, Bull. Amer. Math. Soc., 39, 2002, 355-405.

[25] Gilbarg, D. and Trudinger, N. S., Elliptic Partial Differential Equations of Second Order, 2nd ed., SpringerVerlag, Berlin-New York, 1983.

[26] Gromov, M., Isoperimetric inequalities Riemannian manifolds, asymptotic theory of finite-dimensional normed spaces, Lecture Notes Math., 1200, Appendix I, 114-129, Springer-Verlag, Berlin-New York, 1986.

[27] Hörmander, L., Linear Partial Differential Operators, Springer-Verlag, Berlin-New York, 1969.

[28] Klimov, V. S., On the symmetrization of anisotropic integral functionals, Izv. Vyssh. Uchebn. Zaved. Mat., 99, 1999, 26-32 (in Russian); translation in Russian Math. (Iz. VUZ), 43, 1999, 23-29.

[29] Lions, P.-L. and Pacella, F., Isoperimetric inequality for convex cones, Proc. Amer. Math. Soc., 109, 1990, 477-485.

[30] Milman, E. and Rotem, L., Complemented Brunn-Minkowski inequalities and isoperimetry for homogeneous and non-homogeneous measures, Adv. Math., 262, 2014, 867-908.

[31] Osserman, R., The isoperimetric inequality, Bull. Amer. Math. Soc., 84 1978, 1182-1238.

[32] Serra, J. and Teixidó, M., Isoperimetric inequality in Hadamard manifolds of dimension two via the ABP method, in preparation.

[33] Taylor, J., Existence and structure of solutions to a class of nonelliptic variational problems, Symposia Mathematica, 14, 1974, 499-508.

[34] Taylor, J., Unique structure of solutions to a class of nonelliptic variational problems, Proc. Symp. Pure Math., A. M. S., 27, 1975, 419-427.

[35] Trudinger, N. S., Isoperimetric inequalities for quermassintegrals, Ann. Inst. H. Poincaré Anal. Non Linéaire, 11, 1994, 411-425.

[36] Van Schaftingen, J., Anisotropic symmetrization, Ann. Inst. H. Poincaré Anal. Non Linéaire, 23, 2006, $539-565$.

[37] Wulff, G., Zur frage der geschwindigkeit des wachsturms und der auflösung der kristallflächen, Zeitschrift für Kristallographie, 34, 1901, 449-530. 